\title{
Cognitive full-duplex relay networks under the peak interference power constraint of multiple primary users
}

\author{
Xuan-Toan Doan ${ }^{1}$, Nam-Phong Nguyen ${ }^{1}$, Cheng Yin ${ }^{1}$, Daniel B. da Costa ${ }^{2}$ and Trung Q. Duong ${ }^{1 *}$ (D)
}

\begin{abstract}
This paper investigates the outage performance of cognitive spectrum-sharing multi-relay networks in which the relays operate in a full-duplex (FD) mode and employ the decode-and-forward (DF) protocol. Two relay selection schemes, i.e., partial relay selection (PRS) and optimal relay selection (ORS), are considered to enhance the system performance. New exact expressions for the outage probability (OP) in both schemes are derived based on which an asymptotic analysis is carried out. The results show that the ORS strategy outperforms PRS in terms of OP, and increasing the number of FD relays can significantly improve the system performance. Moreover, novel analytical results provide additional insights for system design. In particular, from the viewpoint of FD concept, the primary network parameters (i.e., peak interference at the primary receivers, number of primary receivers, and their locations) should be carefully considered since they significantly affect the secondary network performance.
\end{abstract}

Keywords: Cognitive radio networks, Full-duplex, Decode-and-forward, Performance analysis

\section{Introduction}

The explosion of data traffic over wireless communication has brought a huge demand for spectrum resources. Cognitive radio has arisen as a promising technology for efficiently utilizing the limited spectrum [1,2]. The key principle behind the functionality of a spectrum sharing approach (also known as underlay strategy) in cognitive radio networks (CRNs) is that unlicensed secondary users are allowed to access the licensed spectrum as long as the interference from the secondary transmitters is harmless to the primary receivers [3]. In order words, the secondary transmitters need to set their transmit powers in order to not cause any interference to the primary network that is above a predefined level. This power adjustment implies a reduction in the coverage area of the secondary network. Fortunately, cooperative relay schemes, which can enhance the coverage of wireless networks by deploying helping nodes to transfer information from the source

*Correspondence: trung.q.duong@qub.ac.uk

This paper has been presented in part at International Conference on Recent Advances on Signal Processing, Telecommunications \& Computing

(SigTelCom), Da Nang, Vietnam, January 2017

${ }^{1}$ Queen's University Belfast, Belfast, UK

Full list of author information is available at the end of the article to the destination, have been introduced in CRNs with spectrum-sharing environment (see, for instance, [4-8]). Specifically, different relay selection schemes assuming amplify-and-forward (AF) and decode-and-forward (DF) relaying protocols, were studied in [6-8], and the results showed that by applying appropriate cooperative schemes, cognitive relay networks can significantly attain improved performance.

However, the aforementioned studies employed halfduplex (HD) relays, which are inefficient from the spectrum usage point of view. Note that a conventional relay uses two time-slots for two operating phases, i.e., listening to messages from the information source node and relaying message to the destination. To tackle this spectral inefficiency, full-duplex (FD) techniques have been proposed for relay networks $[9,10]$, which enable relay nodes to transmit and receive signals simultaneously at the price of self-interference. Although self-interference is an undesirable effect in practice, the developments in signal processing and antenna technologies have proven that FD techniques are a promising solution for efficient spectrum usage in the next generation of wireless communication [11-13]. Moreover, the effect of FD techniques on relay networks has attracted wide attention 
recently [14-16]. The authors in [17-19] investigated a FD, dual-hop, AF system, in which the self-interference variance was modeled as a function of transmit power. In [20], the authors studied different relay selection policies based on the availability of system's channel state information (CSI) at the source node of a multiple AF FD relay system. In [21], motivated by incremental-DF protocol, the authors proposed a new cooperative protocol for FD relays named as incremental-selective-DF and compared its performance with selective-DF protocol in Nakagami$m$ fading environment. In [9], the authors examined the outage probability (OP) of a DF relay network to find the optimal duplex mode of the considered system. However, in all these aforementioned studies, the influence of spectrum-sharing environment on FD relay networks is not well understood. Motivated by this observation, in this paper, we study the performance of cognitive FD relay networks in the presence of multiple FD relays and multiple primary receivers. The contributions of the paper can be summarized as follows:

- Two relay selection schemes, namely, optimal relay selection (ORS) and partial relay selection (PRS), based on the availability of the system CSI at the secondary information source are proposed.

- Due to the existence of the common random variables (RVs), i.e., the channels from the source and/or selected relay to multiple primary receivers, the signal-to-noise-ratios (SNRs) of all links become correlated which makes the analysis troublesome. To get around this challenge, we first apply the conditional probability on these RVs and then derive the analytical expressions for the OP of the considered system. Specifically, the exact and asymptotic expressions of OP in PRS and ORS schemes are also obtained.

- The derived results reveal several insights. For instance, it is shown that the primary network's parameters, i.e., the peak interference constraint, the distance from the primary receivers to the secondary transmitters, and the number of primary receivers, have a high impact on the performance of the secondary network and should be carefully designed. In addition, ORS strategy outperforms PRS 1 in terms of OP, and increasing the number of FD relays can significantly improve the system performance.

The rest of this paper is organized as follows. The system and channel models are introduced in Section 2. Exact and asymptotic expressions for the OP assuming ORS and PRS policies are derived in Sections 3 and 4, respectively. Numerical results are presented in Section 5, which are corroborated through Monte Carlo simulations. Finally, the main conclusions are outlined in Section
6. Appendices A, B, C, and D present the proofs of four Lemmas.

\section{System and channel models}

We consider a cognitive relay network consisting of one secondary transmitter $\mathrm{S}, K$ secondary FD DF relays $\mathrm{R}_{k}$, $k \in\{1, \ldots, K\}$, one secondary receiver $\mathrm{D}$, and $M$ primary receivers $\mathrm{P}_{m}, m \in\{1, \ldots, M\}$, as shown in Fig. 1 . We assume that the primary transmitters are located sufficiently far away from the secondary nodes so as not to impinge any interference upon the received signals at the relays and destination and not to cause perturbation on the relay selection process. The node $S$ is equipped with a single antenna and employs a transmit power $\mathcal{P}_{\mathrm{s}}$. Meanwhile, $\mathrm{R}_{k}$ is equipped with two antennas (one receive antenna and one transmit antenna) for operating in FD mode ${ }^{1}$. All the channels are assumed to experience Rayleigh fading, in which the respective channel power gains are exponentially distributed.

Since a spectrum sharing approach is adopted and $R_{k}$ is in full-duplex mode, the transmit power from $S$ and $R_{k}$ is constrained by the primary network's peak interference parameter $\mathcal{I}_{p}$ as follows:

$$
\mathcal{I}_{p} \geq \mathcal{P}_{\mathrm{S}} \max _{m=1, \ldots, M}\left|h_{\mathrm{SP}_{m}}\right|^{2}+\mathcal{P}_{\mathrm{R}} \max _{m=1, \ldots, M}\left|h_{\mathrm{R}_{k} \mathrm{P}_{m}}\right|^{2}
$$

From (1), non-optimal condition for $\mathcal{P}_{S}$ and $\mathcal{P}_{R}$ can be selected as follows:

$$
\begin{gathered}
\mathcal{P}_{\mathrm{S}}=\frac{\mathcal{I}_{p}}{2 \max _{m=1, \ldots, M}\left|h_{\mathrm{SP}_{m}}\right|^{2}}, \\
\mathcal{P}_{\mathrm{R}}=\frac{\mathcal{I}_{p}}{2 \max _{m=1, \ldots, M} \mid h_{\left.\mathrm{R}_{k} \mathrm{P}_{m}\right|^{2}}},
\end{gathered}
$$

where $\mathcal{P}_{S}$ and $\mathcal{P}_{\mathrm{R}}$ are the transmit powers of $S$ and $R_{k}$, respectively, $h_{\mathrm{SP}_{m}}$ and $h_{\mathrm{R}_{k} \mathrm{P}_{m}}$ denote the channel coefficients pertaining to the $\mathrm{S} \rightarrow \mathrm{P}_{m}$ and $\mathrm{R}_{k} \rightarrow \mathrm{P}_{m}$ links,

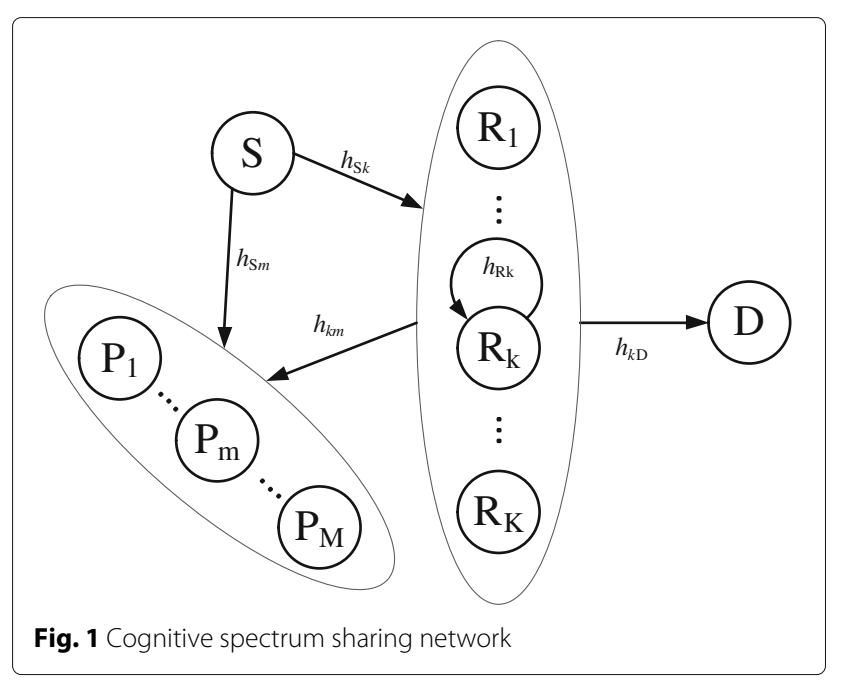


respectively. The CSI of the channel gains from the source and relays to primary users can be obtained from direct feedback from PU-Rx or indirect feedback from band manager [22].

The transmission is performed in two hops. In the first hop, $\mathrm{S}$ transmits information to $\mathrm{R}_{k^{*}}$, where the subscript $k^{*}$ indicates the aiding relay that is chosen in the relay selection process, which will be detailed next. Because $R_{k^{*}}$ simultaneously receives and forwards information, self-interference occurs at the receive antenna of $R_{k^{*}}$. Furthermore, although $R_{k^{*}}$ applies self-interference cancellation techniques, the self-interference channel at $R_{k^{*}}$, i.e., $h_{R_{k^{*}}}$, cannot be fully mitigated and is modeled as an independent Rayleigh distributed channel [23]. In addition, we assume that the impact of interference from the primary transmitter on secondary network is neglected when the primary transmitter is located far away from secondary receiver $[24,25]$. Thus, the received signal at $R_{k^{*}}$ is given by

$$
y_{\mathrm{R}_{k^{*}}}=\sqrt{\mathcal{P}_{\mathrm{S}}} h_{\mathrm{SR}_{\mathrm{k}^{*}} x_{\mathrm{S}}}+\sqrt{\mathcal{P}_{\mathrm{R}}} h_{\mathrm{R}_{k^{*}} x_{\mathrm{R}}}+n_{\mathrm{R}},
$$

where $x_{S}$ and $x_{R}$ represent the transmitted signals from $S$ and $R_{k^{*}}$, respectively, $h_{S R_{k^{*}}}$ is the channel coefficient from $S$ to $R_{k^{*}}$, and $n_{\mathrm{R}} \sim \mathcal{N}\left(0, N_{0}\right)$ is the additive white Gaussian noise (AWGN) at $\mathrm{R}_{k^{*}}$.

After decoding the received information from $S, R_{k^{*}}$ forwards the decoded message to $D$ in the second hop. The received information at $D$ can be expressed as

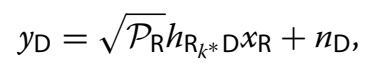

where $h_{\mathrm{R}_{k^{*}} \mathrm{D}}$ denotes the channel coefficient from $\mathrm{R}_{k^{*}}$ to $\mathrm{D}$ and $n_{\mathrm{D}} \sim \mathcal{N}\left(0, N_{0}\right)$ stands for the AWGN term at $\mathrm{D}$.

The signal-to-interference-plus-noise-ratio (SINR) at $\mathrm{R}_{k^{*}}$ and SNR at $\mathrm{D}$ can be written, respectively, as

$$
\begin{aligned}
\gamma_{1 k^{*}} & =\frac{\mathcal{P}_{\mathrm{S}}\left|h_{\mathrm{SR}_{k^{*}}}\right|^{2}}{\mathcal{P}_{\mathrm{R}}\left|h_{\mathrm{R}_{k^{*}}}\right|^{2}+N_{0}}, \\
\gamma_{2 k^{*}} & =\frac{\mathcal{P}_{\mathrm{R}}\left|h_{\mathrm{R}_{k^{*}} \mathrm{D}}\right|^{2}}{N_{0}} .
\end{aligned}
$$

In the considered system, we assume that $R_{k}$ employs a DF protocol thanks to its better performance in the presence of self-interference compared to that of AF protocol [26]. Thus, the end-to-end SNR can be computed as [5]

$$
\gamma_{k^{*}}=\min \left(\gamma_{1 k^{*}}, \gamma_{2 k^{*}}\right) \text {. }
$$

From (8), the capacity of the overall transmission $S \rightarrow$ $\mathrm{R}_{k} \rightarrow \mathrm{D}$ can be expressed as

$$
C=\log _{2}\left[1+\gamma_{k^{*}}\right] \text {. }
$$

Knowing that the OP is defined as the probability that the channel capacity of the considered system falls below a given threshold, such a metric can be formulated as

$$
\mathbb{P}\left\{C<R_{\text {th }}\right\}=\mathbb{P}\left\{\gamma_{k^{*}}<2^{R_{\text {th }}}-1\right\}=F_{\gamma_{k^{*}}}(\beta),
$$

where $\mathbb{P}\{\cdot\}$ denotes probability, $F_{\gamma_{k^{*}}}(\cdot)$ represents the cumulative distribution function (CDF) of $\gamma_{k^{*}}, \beta=2^{R_{\text {th }}}$ 1 , and $R_{\text {th }}$ is the target rate of the secondary network.

\section{Outage probability analysis}

\subsection{Partial relay selection (PRS)}

In some networks, such as wireless sensor networks, the energy and computational resources are limited. Therefore, utilizing aiding relay based on full global CSI is infeasible. Motivated by this fact, in PRS scheme, the node $S$ uses the CSI solely of the channels pertaining to firsthop transmission to select the aiding relay $\mathrm{R}_{p}$ that has the best link from S, i.e.,

$$
k_{\mathrm{PRS}}^{*}=\arg \max _{k=1, \ldots, K}\left(\left|h_{\mathrm{SR}_{k}}\right|^{2}\right) \text {. }
$$

From (2), (3), (6), and (7), the SINR of the first hop and the SNR of the second hop are formulated, respectively, as

$$
\begin{aligned}
\gamma_{1 k^{*}}^{\mathrm{PRS}} & =\frac{\frac{\mathcal{I}_{p}}{2 \max _{m=1, \ldots, M}\left|h_{S \mathrm{P}_{m}}\right|^{2}} \max _{k=1, \ldots, K}\left|h_{\mathrm{SR}_{k}}\right|^{2}}{\left(\frac{\mathcal{I}_{p}}{\max _{m=1, \ldots, M} \mid h_{\left.\mathrm{R}_{p} \mathrm{P}_{m}\right|^{2}}}\left|h_{\mathrm{R}_{p}}\right|^{2}+N_{0}\right)} \\
& =\frac{\frac{\gamma_{1}}{\max _{m=1, \ldots, M}\left|h_{S \mathrm{P}_{m}}\right|^{2}} \max _{k=1, \ldots, K}\left|h_{\mathrm{SR}_{k}}\right|^{2}}{\frac{\gamma_{1}}{\left.\max _{m=1, \ldots, M}\left|h_{\left.\mathrm{R}_{p} \mathrm{P}_{m}\right|^{2}}\right| h_{\mathrm{R}_{p}}\right|^{2}+1}} \\
\gamma_{2 k^{*}}^{\mathrm{PRS}} & =\frac{\mathcal{I}_{p}\left|h_{\mathrm{R}_{p} \mathrm{D}}\right|^{2}}{2 \max _{m=1, \ldots, M}\left|h_{\mathrm{R}_{p} \mathrm{P}_{m}}\right|^{2} N_{0}} \\
& =\frac{\gamma_{1}\left|h_{\mathrm{R}_{p} \mathrm{D}}\right|^{2}}{\max _{m=1, \ldots, M}\left|h_{\mathrm{R}_{p} \mathrm{P}_{m}}\right|^{2}},
\end{aligned}
$$

where $\gamma_{1}=\frac{\mathcal{I}_{p}}{2 N_{0}}$. From (8), (12), and (13), the end-to-end SNR of the considered PRS policy is given by

$$
\gamma_{k^{*}}^{\mathrm{PRS}}=\min \left(\gamma_{1 k^{*}}^{\mathrm{PRS}}, \gamma_{2 k^{*}}^{\mathrm{PRS}}\right) .
$$

From (12)-(14), we have the following lemma.

Lemma 1 The OP of the considered PRS scheme is given as follows:

$$
\begin{aligned}
\mathbb{P}_{\text {out }}^{\mathrm{PRS}}= & 1+\sum_{m_{1}=1}^{M} \sum_{m_{2}=1}^{M} \sum_{k=1}^{K}\left(\begin{array}{c}
M \\
m_{1}
\end{array}\right)\left(\begin{array}{c}
M \\
m_{2}
\end{array}\right)\left(\begin{array}{c}
K \\
k
\end{array}\right) \\
& (-1)^{k+m_{1}+m_{2}} \frac{m_{1} m_{2} \lambda_{\mathrm{R}} \lambda_{\mathrm{RP}} \lambda_{\mathrm{SP}}}{2 \beta k \lambda_{\mathrm{SR}}\left(\frac{\beta \lambda_{\mathrm{RD}}}{\gamma_{1}}+m_{2} \lambda_{\mathrm{RP}}\right)^{2}} \\
& \times{ }_{2} F_{1}\left(1,2 ; 3 ; 1-\left(\frac{1}{\gamma_{1}}+\frac{m_{1} \lambda_{\mathrm{SP}}}{k \beta \lambda_{\mathrm{SR}}}\right) \frac{\lambda_{\mathrm{R}}}{\frac{\lambda_{\mathrm{RD}} \beta}{\gamma_{1}}+\lambda_{\mathrm{RP}} m_{2}}\right)
\end{aligned}
$$


where ${ }_{2} F_{1}(\cdot, \cdot ; \cdot ; \cdot)$ symbolizes the Gaussian hypergeometric function ([27], Eq. (9.14.1)) and $\lambda_{X}^{-1}$ is the mean value of exponential random variable $\left|h_{X}\right|^{2}, X \in$ $\left\{\mathrm{SR}_{k}, \mathrm{R}_{k} \mathrm{D}, \mathrm{SP}_{m}, \mathrm{R}_{k} \mathrm{P}_{m}, \mathrm{R}_{k}\right\}$.

\section{Proof The proof is given in Appendix A.}

\subsection{Optimal relay selection (ORS)}

In ORS scheme, the node $S$ is assumed to be a stationary base station that has rich energy and powerful computational resources. Therefore, $\mathrm{S}$ is able to collect and process all the system CSI to choose the aiding relay that can maximize the capacity of the considered system. In this case, the aiding relay $\mathrm{R}_{o}$ is selected according to the following rule:

$$
k_{\mathrm{ORS}}^{*}=\arg \max _{k=1, \ldots, K} \min \left(\gamma_{1 k}, \gamma_{2 k}\right)
$$

The end-to-end SNR is given by

$$
\gamma_{k^{*}}^{\mathrm{ORS}}=\max _{k=1, \ldots, K} \min \left(\gamma_{1 k}, \gamma_{2 k}\right),
$$

where $\gamma_{1 k}$ and $\gamma_{2 k}$ denote the SINR of the first hop and the SNR of the second hop, respectively, and are expressed as

$$
\begin{aligned}
\gamma_{1 k} & =\frac{\frac{\gamma_{1}}{\max _{m=1, \ldots, M}\left|h_{S P_{m}}\right|^{2}}\left|h_{\mathrm{SR}_{k}}\right|^{2}}{\frac{\gamma_{1}}{\left.\max _{m=1, \ldots, M}\left|h_{\left.\mathrm{R}_{p} \mathrm{P}_{m}\right|^{2}}\right| h_{\mathrm{R}_{p}}\right|^{2}+1},} \\
\gamma_{2 k} & =\frac{\gamma_{1}\left|h_{\mathrm{R}_{k} \mathrm{D}}\right|^{2}}{\max _{m=1, \ldots, M}\left|h_{\mathrm{R}_{k} \mathrm{P}_{m}}\right|^{2}},
\end{aligned}
$$

From (17)-(19), we have the following lemma.

Lemma 2 The OP of the considered ORS scheme is given as follows:

$\mathbb{P}_{\text {out }}^{\mathrm{ORS}}=1-\sum_{n=1}^{M}\left(\begin{array}{c}M \\ n\end{array}\right) n \lambda_{\mathrm{SP}}(-1)^{n+1} \int_{0}^{\infty} \Delta_{1}^{K} \exp (-n \lambda \mathrm{SP} y) d y$,

where

$$
\begin{aligned}
\Delta_{1}= & 1-\sum_{m=1}^{M}\left(\begin{array}{c}
M \\
m
\end{array}\right)(-1)^{m+1} m \lambda_{\mathrm{RP}} \exp \left(-\frac{\beta \lambda_{\mathrm{SR}}}{\gamma_{1}} y\right) \\
& \left\{\frac{\beta \lambda_{\mathrm{SR}}}{\lambda_{\mathrm{R}}} y \exp \left(\frac{\beta \lambda_{\mathrm{SR}}\left(\frac{\beta \lambda_{\mathrm{RD}}}{\gamma_{1}}+m \lambda_{\mathrm{RP}}\right)}{\lambda_{\mathrm{R}}} y\right)\right. \\
& \left.\times E i\left(-\frac{\beta \lambda_{\mathrm{SR}}}{\lambda_{\mathrm{R}}}\left(\frac{\beta \lambda_{\mathrm{RD}}}{\gamma_{1}}+m \lambda_{\mathrm{RP}}\right) y\right)+\frac{\gamma_{1}}{\beta \lambda_{\mathrm{RD}}+m \lambda_{\mathrm{RP}} \gamma_{1}}\right\}
\end{aligned}
$$

Proof The proof is given in Appendix B.

\section{Asymptotic outage analysis}

In this section, an asymptotic outage analysis is carried out in order to gain further insights into the system performance. As will be shown, the considered system has null diversity order because of the self-interference effect inherent to the FD technique ${ }^{2}$. In addition, from the asymptotic expressions, we can observe that in the high SNR regime, the performance of the considered system does not depend on the channel of the second hop. In other words, to enhance the performance of the considered system, the performance of the first hop must be strengthen.

\subsection{Partial relay selection}

Lemma 3 In the high SNR regime, the OP of the considered system with PRS scheme can be expressed as

$$
\begin{gathered}
\mathbb{P}_{\text {out }}^{\text {PRS } \stackrel{\text { high } S N R}{\approx}} 1+\sum_{m_{1}=1}^{M} \sum_{m_{2}=1}^{M} \sum_{k=1}^{K}\left(\begin{array}{c}
M \\
m_{1}
\end{array}\right)\left(\begin{array}{c}
M \\
m_{2}
\end{array}\right)\left(\begin{array}{c}
K \\
k
\end{array}\right) \\
(-1)^{k+m_{1}+m_{2}} \frac{m_{1} m_{2} \lambda_{\mathrm{R}} \lambda_{\mathrm{RP}} \lambda_{\mathrm{SP}}}{2 \beta k \lambda_{\mathrm{SR}}\left(m_{2} \lambda_{\mathrm{RP}}\right)^{2}} \\
\times{ }_{2} F_{1}\left(1,2 ; 3 ; 1-\frac{m_{1} \lambda_{\mathrm{SP}} \lambda_{\mathrm{R}}}{m_{2} k \beta \lambda_{\mathrm{SR}} \lambda_{\mathrm{RP}}}\right) .
\end{gathered}
$$

Proof The proof is given in Appendix C.

\subsection{Optimal relay selection}

Lemma 4 In the high SNR regime, the OP of the considered system with ORS scheme can be expressed as

$$
\begin{aligned}
& \mathbb{P}_{\text {out }}^{\mathrm{ORS}} \stackrel{\text { high SNR }}{\approx} 1-\sum_{n=1}^{M}\left(\begin{array}{c}
M \\
n
\end{array}\right) n \lambda_{\mathrm{SP}}(-1)^{n+1} \int_{0}^{\infty} \\
& \Delta_{2}^{K} \exp \left(-n \lambda_{\mathrm{SP}} y\right) d y \text {, }
\end{aligned}
$$

where

$$
\begin{aligned}
\Delta_{2}= & \sum_{m=1}^{M}\left(\begin{array}{c}
M \\
m
\end{array}\right)(-1)^{m+1} \frac{m \beta \lambda_{\mathrm{SR}} \lambda_{\mathrm{RP}} y}{\lambda_{\mathrm{R}}} \exp \left(\frac{m \beta \lambda_{\mathrm{SR}} \lambda_{\mathrm{RP}}}{\lambda_{\mathrm{R}}} y\right) \\
& \mathbf{E i}\left(-\frac{m \beta \lambda_{\mathrm{SR}} \lambda_{\mathrm{RP}}}{\lambda_{\mathrm{R}}} y\right) .
\end{aligned}
$$

Proof The proof is given in Appendix D.

\section{Numerical results and discussions}

In this section, numerical examples are presented to show the impact of the network's parameters on the overall system performance. The accuracy of our analysis is attested by Monte Carlo simulations, in which a perfect agreement between the analytical and simulated curves 
is observed. Without loss of generality, we adopt a twodimensional topology in which the coordinates $(x, y)$ of $S, R$, and $D$ are $(0,0),(2,0)$, and $(3,0)$, respectively. Thus, the Euclidian distance between the nodes can be calculated as $d_{A B}=\sqrt{\left(x_{A}-x_{B}\right)^{2}+\left(y_{A}-y_{B}\right)^{2}}$, where $A$ is placed at $\left(x_{A}, y_{A}\right)$ and $B$ has the coordinate $\left(x_{B}, y_{B}\right)$. In order to take the path-loss into account, we assume $\lambda_{X}=d_{X}^{-\alpha}$, where $\alpha$ denotes the path-loss exponent and $\lambda_{X} \in\left\{\lambda_{\mathrm{SP}}, \lambda_{\mathrm{SR}}, \lambda_{\mathrm{RP}}, \lambda_{\mathrm{RD}},\right\}$. Without loss of generality, in the plots, we set $\alpha=4$ and the target rate of the secondary network $R_{\text {th }}=0.4 \mathrm{bits} / \mathrm{s} / \mathrm{Hz}$, and $\lambda_{R}=22$.

Figures 2 and 3 show the effect of the primary users' locations on the system OP for the PRS and ORS schemes, respectively. From these figures, one can observe that as the primary receivers move farther from the secondary transmitters, the performance of the secondary network improves. The reason is that the longer the distance between the primary receiver and the secondary transmitter results in the higher the transmit power of the secondary transmitter, which implies a better secondary transmission. In addition, the effects of the primary network's peak interference constraint on the secondary network are also revealed. Note that, if the peak interference constraint is too small, the secondary transmitters will not have sufficient transmit power to ensure good transmissions. On the other hand, if the peak interference constraint is too high, the transmit power at the FD relays will be large, resulting in high levels of self-interference. Besides, these two figures also show that the ORS scheme outperforms PRS one.

In Fig. 4, the effect of the number of primary receivers on system OP is investigated. As can be seen, when the SNR is low, increasing the number of primary

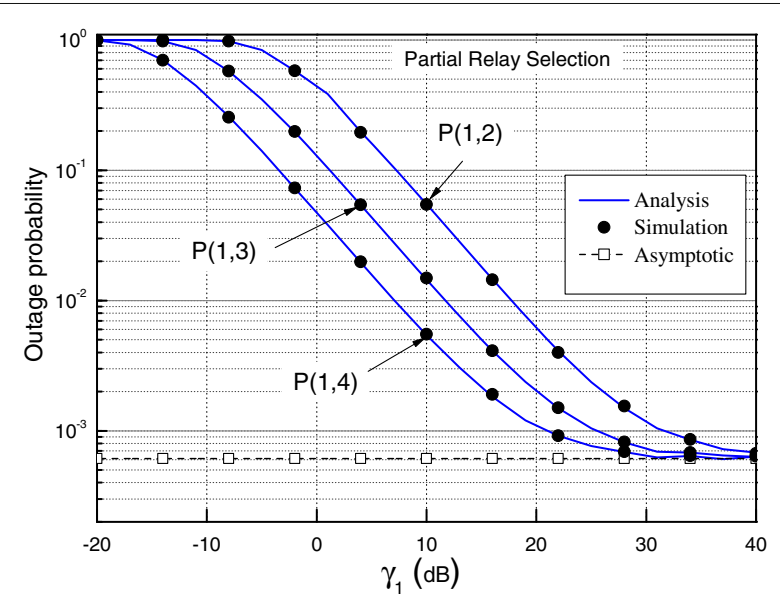

Fig. 2 Effect of primary users' positions on system OP for PRS scheme

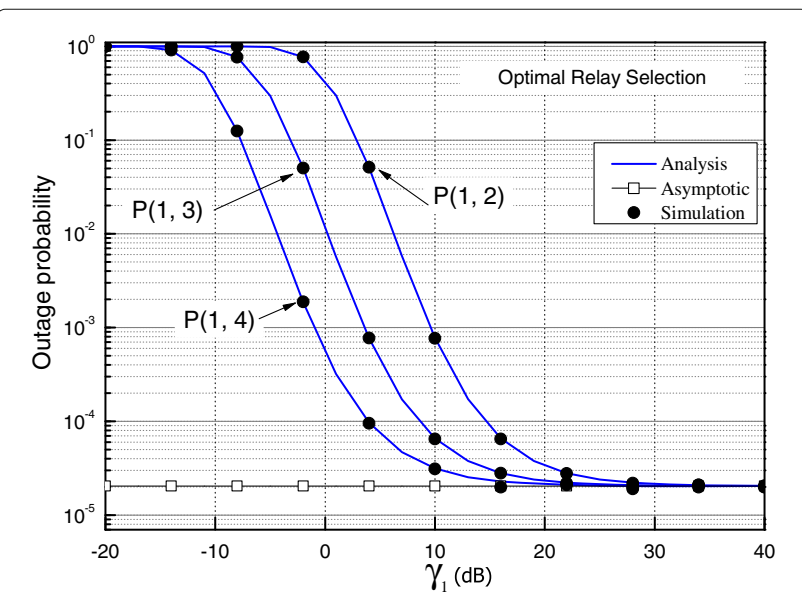

Fig. 3 Effect of primary users' positions on the system OP for ORS scheme

receivers decreases the performance of the considered system. However, at high SNR regions, increasing the number of primary receivers will force the secondary transmitters to reduce their transmit powers, reducing the self-interference at the relay and consequently the OP.

Figures 5 and 6 illustrate the influence of selfinterference on the secondary network's performance by varying the mean power of self-interference channel, i.e., $\frac{1}{\lambda_{R}}$. As expected, the worse the quality of the self-interference channel is, the better the system performance is. Particularly, as the SNR of the considered system is high, the smaller the mean power of self-interference channel is, the higher the system diversity gain is. In addition, the results show that applying ORS scheme can

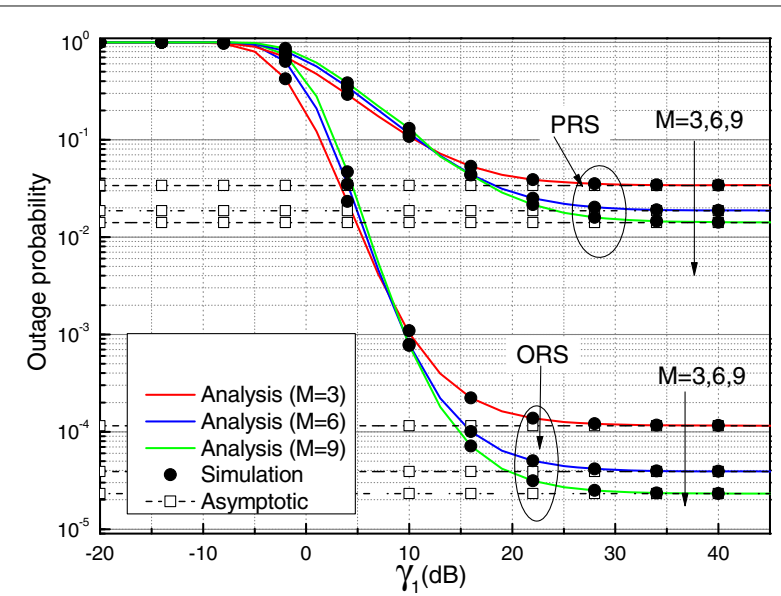

Fig. $4 \mathrm{OP}$ for different numbers of primary receivers 


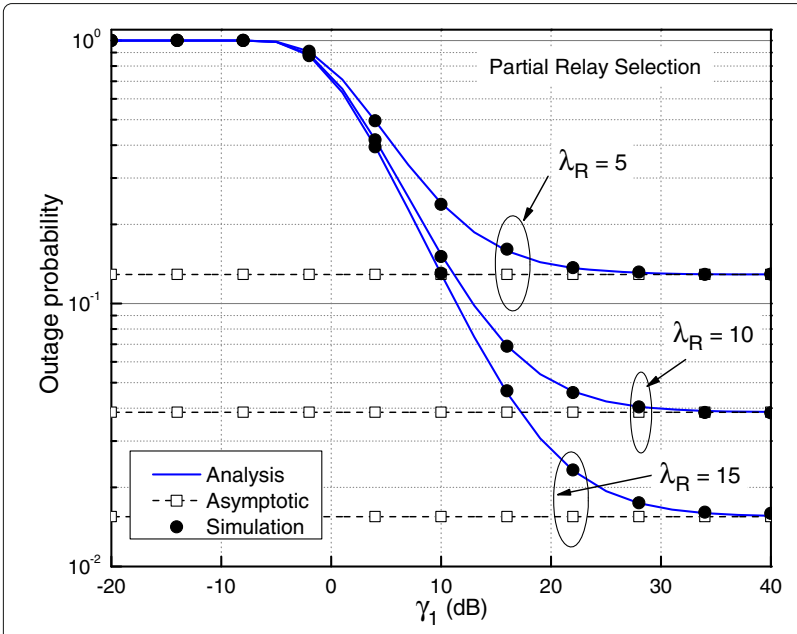

Fig. 5 Effect of self-interference on the system OP with PRS scheme

restraint the effect of self-interference better than PRS scheme.

Figure 7 demonstrates the influence of the number of relays on the system OP. When the number of relays increases, the performance of the considered system is enhanced. Particularly, a more significant improvement can be witnessed in ORS scheme than in PRS scheme.

Figure 8 shows the comparison in system's capacity between FD and HD relays. We can observe that applying FD relays can help the system achieve a higher capacity. The reason is that FD relays can listen and transmit information simultaneously while HD relays have two phases of operation, i.e., listening to the information source and relaying signal to the destination.

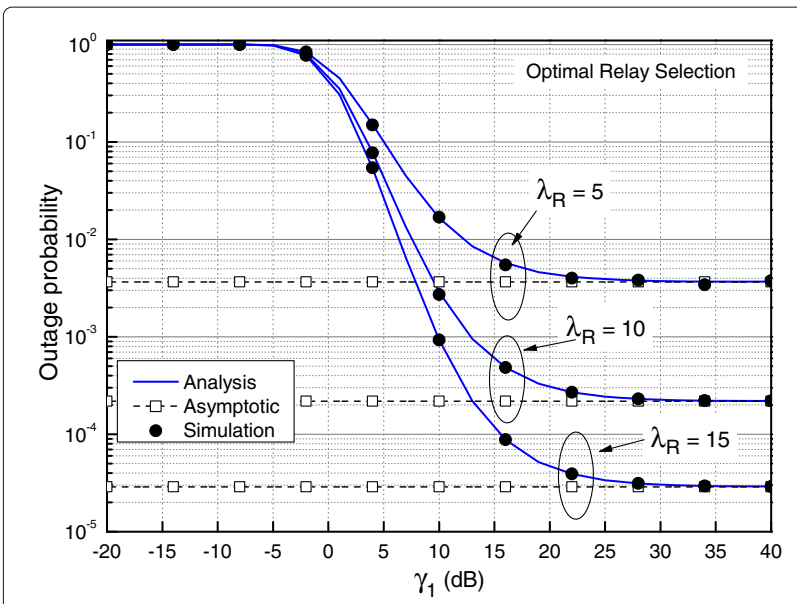

Fig. 6 Effect of self-interference on the system OP with ORS scheme

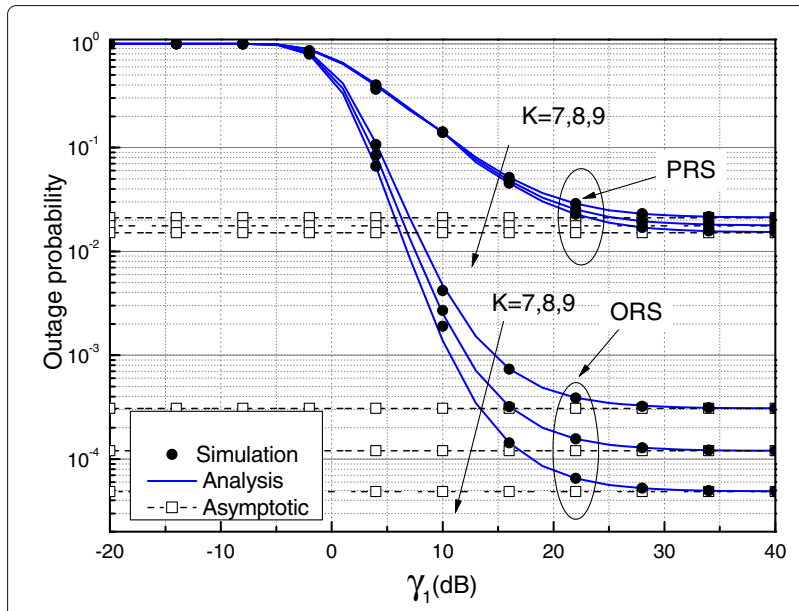

Fig. 7 OP for different numbers of FD relays

\section{Conclusions}

In this paper, the system performance in terms of outage probability of a cognitive FD relay network in the presence of multiple primary receivers has been evaluated. In particular, two relay selection strategies, namely ORS and PRS, have been proposed to enhance the performance of the system. New exact and asymptotic expressions for the OP of the PRS and ORS schemes were derived. The results showed that the ORS scheme has a better performance than PRS scheme. In addition, increasing the number of FD relays can enhance the system performance. Primary network designing parameters, i.e., the peak interference constraint, the number of primary receivers, and their locations, have great influences on the performance of the secondary network and should be mindfully considered.

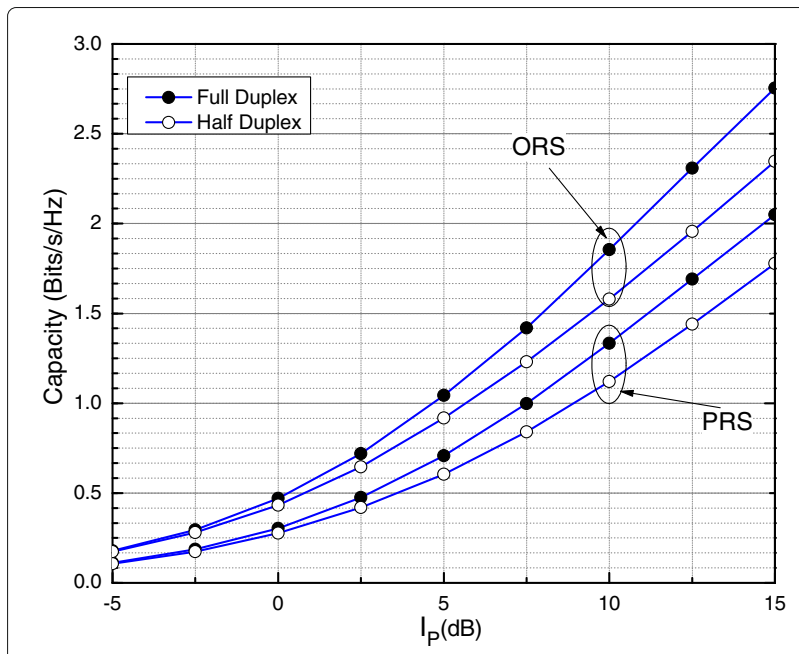

Fig. 8 Comparison between FD and HD relays 


\section{Endnotes}

${ }^{1}$ Dual-antenna FD implementation is one of many ways to deploy FD mode, including single-antenna FD implementation.

${ }^{2}$ In the considered system, the transmit power at the full-duplex relay is non-optimal. A transmit power optimization scheme at full-duplex relay can be considered to restrain the self-interference effect.

\section{Appendix A: Proof of Lemma 1}

Let

$$
\begin{aligned}
& \mathcal{X}_{1}=\max _{k=1, \ldots, K}\left|h_{\mathrm{SR}_{k}}\right|^{2}, \\
& \mathcal{X}_{2}=\max _{m=1, \ldots, M}\left|h_{\mathrm{SP}_{m}}\right|^{2}, \\
& \mathcal{X}_{3}=\left|h_{\mathrm{R}_{p}}\right|^{2}, \\
& \mathcal{X}_{4}=\max _{m=1, \ldots, M}\left|h_{\mathrm{R}_{p} \mathrm{P}_{m}}\right|^{2}, \\
& \mathcal{X}_{5}=\left|h_{\mathrm{R}_{p} \mathrm{D}}\right|^{2} .
\end{aligned}
$$

The CDF and the probability density function (PDF) of $\mathcal{X}_{1}$ can be expressed as

$$
\begin{aligned}
& F_{\mathcal{X}_{1}}(x)=1-\sum_{k=1}^{K}\left(\begin{array}{c}
K \\
k
\end{array}\right)(-1)^{k+1} \exp \left(-k \lambda_{\mathrm{SR}} x\right), \\
& f_{\mathcal{X}_{1}}(x)=\sum_{k=1}^{K}\left(\begin{array}{l}
K \\
k
\end{array}\right)(-1)^{k+1} k \lambda_{\mathrm{SR}} \exp \left(-k \lambda_{\mathrm{SR}} x\right) .
\end{aligned}
$$

In the same way, the CDF and PDF of $\mathcal{X}_{2}$ are given by

$$
\begin{aligned}
& F_{\mathcal{X}_{2}}(x)=1-\sum_{m=1}^{M}\left(\begin{array}{c}
M \\
m
\end{array}\right)(-1)^{m+1} \exp \left(-m \lambda_{\mathrm{SP} x}\right), \\
& f_{\mathcal{X}_{2}}(x)=\sum_{m=1}^{M}\left(\begin{array}{c}
M \\
m
\end{array}\right)(-1)^{m+1} m \lambda_{\mathrm{SR}} \exp \left(-m \lambda_{\mathrm{SP}} x\right),
\end{aligned}
$$

and the CDF and PDF of $\mathcal{X}_{4}$ can be written as

$$
\begin{aligned}
& F_{\mathcal{X}_{4}}(x)=1-\sum_{m=1}^{M}\left(\begin{array}{c}
M \\
m
\end{array}\right)(-1)^{m+1} \exp \left(-m \lambda_{\mathrm{RP}} x\right), \\
& f_{\mathcal{X}_{4}}(x)=\sum_{m=1}^{M}\left(\begin{array}{c}
M \\
m
\end{array}\right)(-1)^{m+1} m \lambda_{\mathrm{RP}} \exp \left(-m \lambda_{\mathrm{RP}} x\right) .
\end{aligned}
$$

Finally, the CDF and PDF of $\mathcal{X}_{3}$ are given by

$$
\begin{aligned}
F_{\mathcal{X}_{3}}(x) & =1-\exp \left(-\lambda_{\mathrm{R}} x\right), \\
f_{\mathcal{X}_{3}}(x) & =\lambda_{\mathrm{R}} \exp \left(-\lambda_{\mathrm{R}} x\right),
\end{aligned}
$$

and the CDF and PDF of $\mathcal{X}_{5}$ are given by

$$
\begin{aligned}
F_{\mathcal{X}_{5}}(x) & =1-\exp \left(-\lambda_{\mathrm{RD}} x\right), \\
f_{\mathcal{X}_{5}}(x) & =\lambda_{\mathrm{RD}} \exp \left(-\lambda_{\mathrm{RD}} x\right) .
\end{aligned}
$$

From (12) and (13), $\gamma_{1 k^{*}}^{\mathrm{PRS}}$ and $\gamma_{2 k^{*}}^{\mathrm{PRS}}$ can be rewritten as follows:

$$
\begin{aligned}
& \gamma_{1 k^{*}}^{\mathrm{PRS}}=\frac{\gamma_{1} \frac{\mathcal{X}_{1}}{\mathcal{X}_{2}}}{\gamma_{1} \frac{\mathcal{X}_{3}}{\mathcal{X}_{4}}+1}, \\
& \gamma_{2 k^{*}}^{\mathrm{PRS}}=\gamma_{1} \frac{\mathcal{X}_{5}}{\mathcal{X}_{4}} .
\end{aligned}
$$

Since $\gamma_{1 k^{*}}^{\mathrm{PRS}}$ and $\gamma_{2 k^{*}}^{\mathrm{PRS}}$ depend on $\mathcal{X}_{4}$, the CDF of $\gamma_{k^{*}}^{\mathrm{PRS}}$ can be expressed as

$$
\begin{aligned}
F_{\gamma_{k^{*}}^{\mathrm{PRS}}}(\beta)= & \int_{0}^{\infty}\left[1-\left(1-F_{\gamma_{k^{*}}^{\mathrm{PRS}} \mid \mathcal{X}_{4}}(\beta)\right)\right. \\
& \left.\left(1-F_{\gamma_{2 k^{*}}^{\mathrm{PPS}} \mid \mathcal{X}_{4}}(\beta)\right)\right] f_{\mathcal{X}_{4}}(x) d x .
\end{aligned}
$$

In order to derive $F_{\gamma_{k^{*}}^{\text {PRS }}}(\beta)$, we need to determine $F_{\gamma_{1 k^{*}}} \mathcal{X}_{4} \beta$ and $F_{\gamma_{2 k^{*}}^{\text {PRS }}} \mid \mathcal{X}_{4}(\beta)$. In this case, $F_{\gamma_{1 k^{*}}^{\text {PRS }}} \mathcal{X}_{4}(\beta)$ can be written as

$$
\begin{aligned}
F_{\gamma_{2 k^{*}}^{\mathrm{PRS}} \mid \mathcal{X}_{4}}(\beta) & =\mathbb{P}\left\{\frac{\gamma_{1} \mathcal{X}_{5}}{\mathcal{X}_{4}}<\beta\right\}=F_{\mathcal{X}_{5}}\left(\frac{\mathcal{X}_{4} \beta}{\gamma_{1}}\right) \\
& =1-\exp \left(-\frac{\lambda_{\mathrm{RD}} \mathcal{X}_{4} \beta}{\gamma_{1}}\right) .
\end{aligned}
$$

Similarly, $F_{\gamma_{1 k^{*}}^{\mathrm{PRS}} \mid \mathcal{X}_{4}}(\beta)$ can be calculated as

$$
\begin{aligned}
F_{\gamma_{1 k^{*}}^{\mathrm{PRS}} \mid \mathcal{X}_{4}}(\beta)= & \mathbb{P}\left\{\frac{\gamma_{1} \frac{\mathcal{X}_{1}}{\mathcal{X}_{2}}}{\gamma_{1} \frac{\mathcal{X}_{3}}{\mathcal{X}_{4}}+1}<\beta\right\} \\
= & \int_{0}^{\infty} \int_{0}^{\infty} F_{\mathcal{X}_{1}}\left[\beta\left(\frac{1}{\gamma_{1}}+\frac{y}{\mathcal{X}_{4}}\right) x\right] f_{\mathcal{X}_{2}}(x) f_{\mathcal{X}_{3}}(y) d x d y, \\
= & 1+\sum_{m_{1}=1}^{M} \sum_{k=1}^{K}(-1)^{m_{1}+k}\left(\begin{array}{c}
M \\
m_{1}
\end{array}\right) \\
& \left(\begin{array}{c}
K \\
k
\end{array}\right) \frac{m_{1} \lambda_{\mathrm{R}} \lambda_{\mathrm{SP}} \mathcal{X}_{4}}{k \beta \lambda_{\mathrm{SR}}} \exp \left[\left(\frac{1}{\gamma_{1}}+\frac{m_{1} \lambda_{\mathrm{SP}}}{k \beta \lambda_{\mathrm{SR}}}\right) D\right] \\
& \times \operatorname{Ei}\left[-\left(\frac{1}{\gamma_{1}}+\frac{m_{1} \lambda_{\mathrm{SP}}}{k \beta \lambda_{\mathrm{SR}}}\right) \mathcal{X}_{4}\right],
\end{aligned}
$$

where $\operatorname{Ei}(\cdot)$ denotes the exponential integral function and (40) is obtained with the help of ([27], Eq. (3.351)). By substituting (39) and (40) into (38), (15) is attained. 


\section{Appendix B: Proof of Lemma 2}

Let

$$
\begin{aligned}
\mathcal{Y}_{1 k} & =\left|h_{\mathrm{SR}_{k}}\right|^{2}, \\
\mathcal{Y}_{3 k} & =\left|h_{\mathrm{R}_{k}}\right|^{2}, \\
\mathcal{Y}_{4 k} & =\max _{m=1, \ldots, M}\left|h_{\mathrm{R}_{k} \mathrm{P}_{m}}\right|^{2}, \\
\mathcal{Y}_{5 k} & =\left|h_{\mathrm{R}_{k} \mathrm{D}}\right|^{2}, \\
\Psi_{k} & =\min \left(\gamma_{1 k}, \gamma_{2 k}\right) .
\end{aligned}
$$

The CDF and PDF of $\mathcal{Y}_{4 k}$ are given by

$$
\begin{aligned}
& F_{\mathcal{Y}_{4 k}}(x)=1-\sum_{m=1}^{M}\left(\begin{array}{c}
M \\
m
\end{array}\right)(-1)^{m+1} \exp \left(-m \lambda_{\mathrm{RP}} x\right), \\
& f_{\mathcal{Y}_{4 k}}(x)=\sum_{m=1}^{M}\left(\begin{array}{c}
M \\
m
\end{array}\right)(-1)^{m+1} m \lambda_{\mathrm{RP}} \exp \left(-m \lambda_{\mathrm{RP}} x\right) .
\end{aligned}
$$

From (18) and (19), $\gamma_{1 k}$ and $\gamma_{2 k}$ can be rewritten as

$$
\begin{aligned}
& \gamma_{1 k}=\frac{\gamma_{1} \frac{\mathcal{Y}_{1 k}}{\mathcal{X}_{2}}}{\gamma_{1} \frac{\mathcal{Y}_{3 k}}{\mathcal{Y}_{4 k}}+1}, \\
& \gamma_{2 k}=\gamma_{1} \frac{\mathcal{Y}_{5 k}}{\mathcal{Y}_{4 k}} .
\end{aligned}
$$

Since $\Psi_{k}$, for $k \in\{1, \ldots, K\}$, depends on $\mathcal{X}_{2}$, the CDF of $\gamma_{k^{*}}^{\mathrm{ORS}}$ can be expressed as

$$
F_{\gamma_{k^{*}}} \operatorname{ORS}(\beta)=\int_{0}^{\infty}\left[F_{\Psi_{k} \mid \mathcal{X}_{2}}(\beta)\right]^{K} f_{\mathcal{X}_{2}}(y) d y,
$$

From (43) and (44), note that $\gamma_{1 k}$ and $\gamma_{2 k}$ depend on $\mathcal{Y}_{4 k}$. Therefore, the CDF of $\Psi_{k}$ conditioned on $\mathcal{X}_{2}$ can be calculated by

$$
\begin{aligned}
F_{\Psi_{k} \mid \mathcal{X}_{2}}(\beta)= & \int_{0}^{\infty}\left[1-\left(1-F_{\gamma_{1 k} \mid \mathcal{X}_{2}, \mathcal{Y}_{4 k}}(\beta)\right)\right. \\
& \left.\left(1-F_{\gamma_{2 k} \mid \mathcal{Y}_{4 k}}(\beta)\right)\right] f_{\mathcal{Y}_{4 k}}(x) d x .
\end{aligned}
$$

On the other hand, the CDF of $\gamma_{2 k}$ conditioned on $\mathcal{Y}_{4 k}$ can be calculated as

$$
\begin{aligned}
F_{\gamma_{2 k} \mid \mathcal{Y}_{4 k}}(\beta) & =\mathbb{P}\left\{\frac{\gamma_{1} \mathcal{Y}_{5 k}}{\mathcal{Y}_{4 k}}<\beta\right\} \\
& =\mathbb{P}\left\{\mathcal{Y}_{5 k}<\frac{\beta \mathcal{Y}_{4 k}}{\gamma_{1}}\right\} \\
& =F_{\mathcal{Y}_{5 k}}\left(\frac{\beta \mathcal{Y}_{4 k}}{\gamma_{1}}\right) \\
& =1-\exp \left(-\frac{\beta \lambda_{\mathrm{RD}} \mathcal{Y}_{4 k}}{\gamma_{1}}\right),
\end{aligned}
$$

Finally, the CDF of $\gamma_{1 k}$ conditioned on $\mathcal{X}_{2}$ and $\mathcal{Y}_{4 k}$ can be calculated as

$$
\begin{aligned}
& F_{\gamma_{1 k} \mid \mathcal{X}_{2}, \mathcal{Y}_{4 k}}(\beta)=\mathbb{P}\left\{\frac{\frac{\mathcal{Y}_{1 k}}{\mathcal{X}_{2}}}{\frac{\mathcal{Y}_{3 k}}{\mathcal{Y}_{4 k}}+\frac{1}{\gamma_{1}}}<\beta\right\} \\
& =\mathbb{P}\left\{\mathcal{Y}_{1 k}<\beta\left(\frac{\mathcal{Y}_{3 k}}{\mathcal{Y}_{4 k}}+\frac{1}{\gamma_{1}}\right) \mathcal{X}_{2}\right\} \\
& =\int_{0}^{\infty} F_{\mathcal{Y}_{1 k} \mid} \mid \mathcal{Y}_{4 k}\left(\left(\frac{y}{\mathcal{Y}_{4 k}}+\frac{1}{\gamma_{1}}\right) \mathcal{X}_{2} \beta\right) f_{\mathcal{Y}_{3 k}}(y) d y \\
& =1-\frac{\lambda_{\mathrm{R}}}{\lambda_{\mathrm{R}}+\beta \lambda_{\mathrm{SR}} \frac{\mathcal{Y}_{2 k}}{\mathcal{Y}_{4 k}}} \exp \left(-\frac{\beta \lambda_{\mathrm{SR}} \mathcal{Y}_{2 k}}{\gamma_{1}}\right) \text {, }
\end{aligned}
$$

where $F_{\mathcal{Y}_{1 k}}(x)=1-\exp \left(-\lambda_{\mathrm{SR}} x\right)$ and $f_{\mathcal{Y}_{3 k}}(x)=\lambda_{\mathrm{R}} \exp \left(-\lambda_{\mathrm{R}} x\right)$.

Thus, from (48), (47), and (42), the CDF of $\Psi_{k}$ conditioned on $\mathcal{X}_{2}$ can be calculated as

$$
\begin{aligned}
F_{\Psi_{k} \mid \mathcal{X}_{2}}(\beta)= & 1-\int_{0}^{\infty} \sum_{m=1}^{M}\left(\begin{array}{c}
M \\
m
\end{array}\right) \frac{(-1)^{m+1} m \lambda_{\mathrm{RP}} \lambda_{\mathrm{R}}}{\lambda_{\mathrm{R}}+\beta \lambda_{\mathrm{SR}} \frac{\mathcal{X}_{2}}{x}} \\
& \exp \left(-\frac{\beta \lambda_{\mathrm{SR}} \mathcal{X}_{2}}{\gamma_{1}}\right) \exp \left(-\frac{\beta \lambda_{\mathrm{RD}} x}{\gamma_{1}}-m \lambda_{\mathrm{RP}} x\right) d x \\
= & 1-\sum_{m=1}^{M}\left(\begin{array}{c}
M \\
m
\end{array}\right)(-1)^{m+1} m \lambda_{\mathrm{RP}} \exp \left(-\frac{\beta \lambda_{\mathrm{SR}}}{\gamma_{1}} \mathcal{X}_{2}\right) \\
& \left\{\frac{\beta \lambda_{\mathrm{SR}}}{\lambda_{\mathrm{R}}} \mathcal{X}_{2} \exp \left(\frac{\beta \lambda_{\mathrm{SR}}\left(\frac{\beta \lambda_{\mathrm{RD}}}{\gamma_{1}}+m \lambda_{\mathrm{RP}}\right)}{\lambda_{\mathrm{R}}} \mathcal{X}_{2}\right)\right. \\
& \times \operatorname{Ei}\left(-\frac{\beta \lambda_{\mathrm{SR}}}{\lambda_{\mathrm{R}}}\left(\frac{\beta \lambda_{\mathrm{RD}}}{\gamma_{1}}+m \lambda_{\mathrm{RP}}\right) \mathcal{X}_{2}\right) \\
& \left.+\frac{\gamma_{1}}{\beta \lambda_{\mathrm{RD}}+m \lambda_{\mathrm{RP}} \gamma_{1}}\right\}
\end{aligned}
$$

where (49) is obtained with the help of ([27], Eq. (3.352.4)). Now, by substituting (49) and

$$
f_{\mathcal{X}_{2}}(x)=\sum_{m=1}^{M}\left(\begin{array}{c}
M \\
m
\end{array}\right)(-1)^{m+1} m \lambda_{\mathrm{SR}} \exp \left(-m \lambda_{\mathrm{SP} X}\right)
$$

into (45), (20) is attained.

\section{Appendix C: Proof of Lemma 3}

At high SNR regions, i.e., assuming high $\gamma_{1}$, (18) can be rewritten as

$$
\gamma_{1 k^{*}}^{\mathrm{PRS}}=\frac{\gamma_{1} \frac{\mathcal{X}_{1}}{\gamma_{2}}}{\gamma_{1} \frac{\mathcal{X}_{3}}{\mathcal{X}_{4}}+1} \stackrel{\text { large }}{\approx} \gamma_{1} \frac{\mathcal{X}_{1} \mathcal{X}_{4}}{\mathcal{X}_{2} \mathcal{X}_{3}} .
$$


Thus, the CDF of $\gamma_{1 k^{*}}^{\text {PRS }}$ conditioned on $\mathcal{X}_{4}$ is given by

$$
\begin{aligned}
F_{\gamma_{1 k^{*}}^{\mathrm{PRS}} \mid \mathcal{X}_{4}}(\beta)= & 1+\sum_{m_{1}=1}^{M} \sum_{k=1}^{K}(-1)^{m_{1}+k}\left(\begin{array}{c}
M \\
m_{1}
\end{array}\right)\left(\begin{array}{c}
K \\
k
\end{array}\right) \\
& \frac{m_{1} \lambda_{\mathrm{R}} \lambda_{\mathrm{SP}} \mathcal{X}_{4}}{k \beta \lambda_{\mathrm{SR}}} \exp \left(\frac{m_{1} \lambda_{\mathrm{SP}}}{k \beta \lambda_{\mathrm{SR}}} \mathcal{X}_{4}\right) \operatorname{Ei}\left(-\frac{m_{1} \lambda_{\mathrm{SP}}}{k \beta \lambda_{\mathrm{SR}}} \mathcal{X}_{4}\right)
\end{aligned}
$$

Now, analyzing $F_{\gamma_{2 k^{*}}^{\mathrm{PRS}} \mid \mathcal{X}_{4}}(\beta)$ at high $\gamma_{1}$, we have that

$$
F_{\gamma_{2 k^{*}}^{\mathrm{PRS}} \mid \mathcal{X}_{4}}(\beta)=1-\exp \left(-\frac{\lambda_{\mathrm{RD}} \mathcal{X}_{4} \beta}{\gamma_{1}}\right), \stackrel{(a)}{\approx} \frac{\lambda_{\mathrm{RD}} \mathcal{X}_{4} \beta}{\gamma_{1}},
$$

where step (a) is obtained by using the McLaurin expansion of $\exp (x)$ and neglecting the high order items for small $x$.

Making use of the above results, it can be shown that the CDF of $\gamma_{k^{*}}^{\text {PRS }}$ is given by

$$
F_{\gamma_{k^{*}}^{\mathrm{PRS}} \mid \mathcal{X}_{4}}(x)=1-\left[1-F_{\gamma_{1 k^{*}}^{\mathrm{PRS}} \mid \mathcal{X}_{4}}(x)\right]\left[F_{\gamma_{2 k^{*}}^{\mathrm{PRS}} \mid \mathcal{X}_{4}}(x)\right] \stackrel{(b)}{\approx} F_{\gamma_{1 k^{*}}^{\mathrm{PRS}} \mid \mathcal{X}_{4}}(x),
$$

where step $(b)$ is performed by neglecting the high order terms. By substituting (51) into (53), and after some mathematical manipulations, (22) is obtained.

\section{Appendix D: Proof of Lemma 4}

For high $\gamma_{1}, F_{\gamma_{2 k}}\left|\mathcal{X}_{2}\right| \mathcal{Y}_{4 k}$ and $F_{\gamma_{1 k}\left|\mathcal{X}_{2}\right| \mathcal{Y}_{4 k}}$ can be rewritten as follows:

$$
\begin{aligned}
F_{\gamma_{2 k} \mid \mathcal{Y}_{4 k}}(\beta) & =1-\exp \left(-\frac{\beta \lambda_{\mathrm{RD}} \mathcal{Y}_{4 k}}{\gamma_{1}}\right) \\
& \stackrel{(c)}{\approx} \frac{\beta \lambda_{\mathrm{RD}} \mathcal{Y}_{4 k}}{\gamma_{1}}, \\
F_{\gamma_{1 k} \mid \mathcal{X}_{2}, \mathcal{Y}_{4 k}}(\beta) & =1-\frac{\lambda_{\mathrm{R}}}{\lambda_{\mathrm{R}}+\beta \lambda_{\mathrm{SR}} \frac{\mathcal{Y}_{2 k}}{\mathcal{Y}_{4 k}}} \exp \left(-\frac{\beta \lambda_{\mathrm{SR}} \mathcal{Y}_{2 k}}{\gamma_{1}}\right) \\
& \stackrel{(d)}{\approx} 1-\frac{\lambda_{\mathrm{R}}}{\lambda_{\mathrm{R}}+\beta \lambda_{\mathrm{SR}} \frac{\mathcal{Y}_{2 k}}{\mathcal{Y}_{4 k}}},
\end{aligned}
$$

where steps (c) and (d) are performed by using the McLaurin expansion of $\exp (x)$ and neglecting the high order terms for small $x$.

In addition, the CDF of $\Psi_{k}$ conditioned on $\mathcal{X}_{2}$ and $\mathcal{Y}_{4 k}$ can be formulated as

$$
\begin{aligned}
F_{\Psi_{k} \mid \mathcal{X}_{2}, \mathcal{Y}_{4 k}}(\beta) & =1-\left[1-F_{\gamma_{1 k} \mid \mathcal{X}_{2}, \mathcal{Y}_{4 k}}(\beta)\right]\left[1-F_{\gamma_{2 k} \mid \mathcal{Y}_{4 k}}(\beta)\right] \\
& \stackrel{(e)}{\approx} F_{\gamma_{1 k} \mid \mathcal{X}_{2}, \mathcal{Y}_{4 k}}(\beta),
\end{aligned}
$$

where step $(e)$ is performed by neglecting the high order terms. From (55) and (56), the CDF of $\Psi_{k}$ conditioned on $\mathcal{X}_{2}$ can be found as

$$
\begin{aligned}
F_{\Psi_{k} \mid \mathcal{X}_{2}}(\beta)= & \sum_{m=1}^{M}\left(\begin{array}{c}
M \\
m
\end{array}\right)(-1)^{m+1} \frac{m \beta \lambda_{\mathrm{SR}} \lambda_{\mathrm{RP}}}{\lambda_{\mathrm{R}}} \mathcal{X}_{2} \\
& \exp \left(\frac{m \beta \lambda_{\mathrm{SR}} \lambda_{\mathrm{RP}}}{\lambda_{\mathrm{R}}} \mathcal{X}_{2}\right) \operatorname{Ei}\left(-\frac{m \beta \lambda_{\mathrm{SR}} \lambda_{\mathrm{RP}}}{\lambda_{\mathrm{R}}} \mathcal{X}_{2}\right) .
\end{aligned}
$$

Finally, by replacing (57) and

$$
f_{\mathcal{X}_{2}}(y)=\sum_{n=1}^{M}(-1)^{n+1}\left(\begin{array}{c}
M \\
n
\end{array}\right) n \lambda_{\mathrm{SP}} \exp \left(-n \lambda_{\mathrm{SP}} y\right)
$$

into (45), (23) is obtained.

\section{Acknowledgements}

This work was supported by the Newton Institutional Link under Grant ID 172719890 .

\section{Competing interests}

The authors declare that they have no competing interests.

\section{Author details}

${ }^{1}$ Queen's University Belfast, Belfast, UK. ²Department of Computer Engineering, Federal University of Ceará, Sobral, Brazil.

Received: 12 June 2016 Accepted: 12 December 2016

Published online: 05 January 2017

\section{References}

1. J Mitola, GQ Maguire, Cognitive radio: making software radios more personal. IEEE Pers. Commun. 6(4), 13-18 (1999)

2. KJ Kim, TQ Duong, HV Poor, Performance analysis of cyclic prefixed single-carrier cognitive amplify-and-forward relay systems. IEEE Trans. Wireless Commun. 12(1), 195-205 (2013)

3. A Goldsmith, S Jafar, I Maric, S Srinivasa, Breaking spectrum gridlock with cognitive radios: an information theoretic perspective. Proc. IEEE. 97(5), 894-914 (2009)

4. VNQ Bao, TQ Duong, DB da Costa, GC Alexandropoulos, A Nallanathan, Cognitive amplify-and-forward relaying with best relay selection in non-identical Rayleigh fading. IEEE Commun. Lett. 17(3), 475-478 (2013)

5. KJ Kim, TQ Duong, X-N Tran, Performance analysis of cyclic prefixed single-carrier spectrum sharing systems with best relay selection. IEEE Trans. Signal Process. 60(12), 6729-6734 (2012)

6. Y Yang Yan, J Jianwei Huang, J Jing Wang, Dynamic bargaining for relay-based cooperative spectrum sharing. IEEE J. Sel. Areas Commun. 31(8), 1480-1493 (2013)

7. TQ Duong, DB da Costa, TA Tsiftsis, C Zhong, A Nallanathan, Outage and diversity of cognitive relaying systems under spectrum sharing environments in Nakagami-m fading. IEEE Commun. Lett. 16(12), 2075-2078 (2012)

8. TDuong, V Bao, H-J Zepernick, Exact outage probability of cognitive AF relaying with underlay spectrum sharing. IET Electronics Lett. 47(17), 1001 (2011)

9. TKwon, S Lim, S Choi, D Hong, Optimal duplex mode for df relay in terms of the outage probability. IEEE Trans. Veh. Technol. 59(7), 3628-3634 (2010)

10. I Krikidis, HA Suraweera, S Yang, K Berberidis, Full-duplex relaying over block fading channel: a diversity perspective. IEEE Trans. Wireless Commun. 11(12), 4524-4535 (2012)

11. TRiihonen, S Werner, R Wichman, Mitigation of loopback self-interference in full-duplex MIMO relays. IEEE Trans. Signal Process. 59(12), 5983-5993 (2011)

12. D Kim, H Lee, D Hong, A survey of in-band full-duplex transmission: from the perspective of PHY and MAC layers. IEEE Commun. Surv. Tuts. 17(4), 2017-2046 (2015)

13. J Zhou, T-H Chuang, T Dinc, H Krishnaswamy, Integrated wideband self-interference cancellation in the RF domain for FDD and full-duplex wireless. IEEE J. Solid-State Circ. 50(12), 3015-3031 (2015) 
14. HA Suraweera, I Krikidis, G Zheng, C Yuen, PJ Smith, Low-complexity end-to-end performance optimization in MIMO full-duplex relay systems. IEEE Trans. Wireless Commun. 13(2), 913-927 (2014)

15. M Mohammadi, HA Suraweera, Y Cao, I Krikidis, C Tellambura, Full-duplex radio for uplink/downlink wireless access with spatially random nodes. IEEE Trans. Commun. 63(12), 5250-5266 (2015)

16. M Mohammadi, BK Chalise, HA Suraweera, C Zhong, G Zheng, I Krikidis, Throughput analysis and optimization of wireless-powered multiple antenna full-duplex relay systems. IEEE Trans. Commun. 64(4), 1769-1785 (2016)

17. NH Tran, L Jimenez Rodriguez, T Le-Ngoc, Optimal power control and error performance for full-duplex dual-hop AF relaying under residual self-interference. IEEE Commun. Lett. 19(2), 291-294 (2015)

18. LJ Rodriguez, NH Tran, T Le-Ngoc, Performance of full-duplex AF relaying in the presence of residual self-interference. IEEE J. Sel. Areas Commun. 32(9), 1752-1764 (2014)

19. LJ Rodriguez, NH Tran, T Le-Ngoc, Optimal power allocation and capacity of full-duplex AF relaying under residual self-interference. IEEE Wireless Commun. Lett. 3(2), 233-236 (2014)

20. I Krikidis, HA Suraweera, PJ Smith, C Yuen, Full-duplex relay selection for amplify-and-forward cooperative networks. IEEE Trans. Wireless Commun. 11(12), 4381-4393 (2012)

21. MG Khafagy, A Ismail, M-S Alouini, S Aissa, Efficient cooperative protocols for full-duplex relaying over Nakagami- $m$ fading channels. IEEE Trans. Wireless Commun. 14(6), 3456-3470 (2015)

22. J Peha, Approaches to spectrum sharing. IEEE Commun. Mag. 43(2), 10-12 (2005)

23. G Chen, Y Gong, P Xiao, JA Chambers, Physical layer network security in the full-duplex relay system. IEEE Trans. Inf. Forensics Secur. 10(3), 574-583 (2015)

24. KJ Kim, TQ Duong, M Elkashlan, PL Yeoh, HV Poor, MH Lee, Spectrum sharing single-carrier in the presence of multiple licensed receivers. IEEE Trans. Wireless Commun. 12(10), 5223-5235 (2013)

25. KJ Kim, TQ Duong, HV Poor, Outage probability of single-carrier cooperative spectrum sharing systems with decode-and-forward relaying and selection combining. IEEE Trans. Wireless Commun. 12(2), 806-817 (2013)

26. DWK Ng, ES Lo, R Schober, Dynamic resource allocation in MIMO-OFDMA systems with full-duplex and hybrid relaying. IEEE Trans. Commun. 60(5), 1291-1304 (2012)

27. IS Gradshteyn, IM Ryzhik, Table of integrals, series, and products, 7th edn. (Academic press, San Diego, 2007)

\section{Submit your manuscript to a SpringerOpen ${ }^{\circ}$ journal and benefit from:}

- Convenient online submission

- Rigorous peer review

- Immediate publication on acceptance

- Open access: articles freely available online

- High visibility within the field

- Retaining the copyright to your article

Submit your next manuscript at springeropen.com 